\title{
Mean field effects in hot compressed nuclear matter
}

\author{
J. J. Molitoris \\ Department of Physics, Muhlenberg College, Allentown, Pennsylvania 18104 \\ A. Bonasera* and B. L. Winer ${ }^{\dagger}$ \\ Department of Physics, Michigan State University, E. Lansing, Michigan 48824 \\ Horst Stoecker \\ Institut für Theoretische Physik der Johann Wolfgang Goethe Universitat, \\ D-6000 Frankfurt, Federal Republic of Germany \\ (Received 17 October 1986; revised manuscript received 29 October 1987)
}

\begin{abstract}
We study effects of the mean field in hot compressed nuclear matter in the context of the Vlasov Uehling-Uhlenbeck theory. The expansion of a spherical distribution at different temperatures is studied along with collisions of $\mathrm{Nb}+\mathrm{Nb}$ and $\mathrm{Au}+\mathrm{Au}$ at lab energies from 50 to 1050 $\mathrm{MeV} /$ nucleon. In both the expansion and the actual heavy ion collision simulation, a transition behavior is seen only at the lowest temperature $(T<10 \mathrm{MeV})$ or bombarding energy $(E=50$ $\mathrm{MeV} /$ nucleon), where the attractive part of the mean field is able to bind the expanding matter. At the lowest energy one thus sees the formation of a central residue, whereas at higher bombarding energies there is complete disintegration of the centrally colliding nuclei. The spectrum of emitted nucleons is found to be much hotter than the kinetic energy spectrum of the central emitting region. The extracted temperature slope parameters are in agreement with recent data.
\end{abstract}

The idea of a liquid gas phase transition in nuclear physics has been discussed by many authors, both theoretically and experimentally. The models used include hydrodynamic and thermodynamic ${ }^{1-6}$ applications of the Fisher drop model, ${ }^{7}$ a field theoretic model, ${ }^{8}$ and percolation theory. ${ }^{9}$

Nuclear matter, like a van der Waals gas, consists of particles interacting with repulsive cores and long range attractive forces. The nature of the nuclear force as revealed in nucleon-nucleon scattering is rather complex. To study the many body system one thus makes approximations such as that of the nuclear fluid. In the fluid dynamic model, the pressure-density diagram ( $T=$ const) of infinite nuclear matter exhibits the maximum-minimum structure typical for matter with long range attraction and short range repulsion. ${ }^{2}$ The nuclear equation of state exhibits a critical point at $\rho_{c}=0.4 \rho_{0}$ and $T_{c}=18 \mathrm{MeV}$, depending on the type of interactions assumed and on the details of the model. The critical temperature is lower with a softer equation of state. ${ }^{14}$

Phase transitions only rigorously occur in this thermodynamic limit with an infinite number of particles. The finite system may have a critical neighborhood rather than a critical point. ${ }^{10}$ For example, the finite system specific heat has a large peak at $T_{c}$ whereas the infinite system has a sharp singularity. ${ }^{11}$ The critical temperature and density are also lowered in the finite size system: the inclusion of Coulomb and surface effects is expected to lower the critical temperature. ${ }^{10}$

Physically, in a nucleus-nucleus collision, a high density and temperature interaction zone is formed in central events. The expansion of this hot matter can lead to lower densities and possibly a liquid gas phase transition.
The study of fragment yields thus offers the possibility of studying the nuclear equation of state at higher temperatures and lower densities than the ground state. The usual first order phase transition (a discontinuous change in one or more of the first derivatives of the relevant thermodynamic potentials) requires slow enough processes for there to be equilibrium across a phase boundary. This is distinct from the fragmentation induced by ultrarelativistic protons ${ }^{12}$ where the density does not vary much from the ground state density.

The task of observing and understanding such a phase transition is not clear cut. For example, if the temperature of a decidedly macroscopic substance-like water is lowered, then it may be advantageous for clusters of molecules to combine into droplets and for the droplets to combine further. A macroscopic droplet then represents the liquid phase and the critical point is where the differences between the two distinct phases vanish. ${ }^{7}$ However, for the nuclear world, this distinction between microscopic and macroscopic does not exist so clearly. We do not yet know if it makes sense to speak of a nuclear gas and liquid and the formation of droplets for a relatively small number of quantum mechanical nucleons. It is thus a challenging experimental task to find unambiguous signatures of such a nuclear phase transition.

Experimentally, it was first proposed that in analogy to the Fisher liquid drop model, ${ }^{7}$ there might be a critical exponent $\tau$ which could be extracted from the fragment distributions. ${ }^{13-15}$ Recall the theoretical reason for such a critical exponent. The probability for the formation of an $A$ cluster in the Fisher model depends exponentially on the cluster Helmholtz free energy $F_{A}$ and on the chemical potential $\mu:^{7,12}$ 


$$
P(A) \propto A^{-\tau} e^{-\left(F_{A}-\mu A\right) / T},
$$

where $\tau \geq 1$ depends principally on the dimensionality. The rate of falloff of the fragment distribution changes with the temperature. At the critical point, the terms in the exponential cancel leaving only a power law., ${ }^{72}$

Note that experimentally an apparent exponent $\tau^{\prime}-$ which does vary with temperature since it must include the effects of the exponential in Eq. (1)-was extracted from fitting the fragment distributions (see Panagiotou et al. in Ref. 14). The early experimental claim ${ }^{14}$ of a possible nuclear critical temperature $T_{c}=10 \mathrm{MeV}$ is now cast in doubt by more complete data and problems with the treatment of the data. ${ }^{16}$

The mechanism of complex fragment production in high energy heavy ion collisions is thus of interest: Is a liquid gas phase transition a possible mechanism? The questions of equilibration and entropy production are related to this fragmentation problem. Fluid dynamical models assume an arbitrary freeze out at a time $t^{*}$ where the density falls to half of the normal density. Then the light fragment composition is determined from a statistical model ${ }^{17}$ by assuming that the baryon number and energy per particle of the interacting nuclear fluid are conserved. We do not know, however, that the mean free path of nucleons is short enough for fluid models to be applicable. In fact, one estimate from the VlasovUehling-Uhlenbeck approach ${ }^{20}$ finds a large mean free path $(2.6 \mathrm{fm})$ at $85 \mathrm{MeV} /$ nucleon. $^{18}$ Another class of model, the intranuclear cascade, ${ }^{19}$ generally lacks binding and so is unable to dynamically describe the formation of fragments.

Finite size effects and binding are essential to this problem. So we use here the Vlasov-Uehling-Uhlenbeck (VUU) approach which includes the mean field, Pauli blocking, and two-body collisions on a microscopic level. $^{20}$ In the VUU model, one approximately solves the equation

$$
\begin{aligned}
\frac{\partial f}{\partial t}+\mathbf{v} \cdot \frac{\partial f}{\partial \mathbf{r}}-\nabla U \cdot \frac{\partial f}{\partial \mathbf{p}}=1 /(2 m) \int & d^{3} p_{2} d^{3} p_{1}^{\prime} d^{3} p_{2}^{\prime} w\left[f_{1}^{\prime} f_{2}^{\prime}(1-f)\left(1-f_{2}\right)-f f_{2}\left(1-f_{1}^{\prime}\right)\left(1-f_{2}^{\prime}\right)\right] \\
& \times \delta^{3}\left(\mathbf{p}+\mathbf{p}_{2}-\mathbf{p}_{1}^{\prime}-\mathbf{p}_{2}^{\prime}\right) \delta\left(E+E_{2}-E_{1}^{\prime}-E_{2}^{\prime}\right)
\end{aligned}
$$

in a Monte Carlo framework. The appropriate transition probability $w=w\left(\mathbf{p}, \mathbf{p}_{2}, \mathbf{p}_{1}^{\prime}, \mathbf{p}_{2}^{\prime}\right)$ is the free transition matrix $\left|\left\langle\mathbf{p}, \mathbf{p}_{2}|T| \mathbf{p}_{1}^{\prime}, \mathbf{p}_{2}^{\prime}\right\rangle\right|^{2}$ because of the strength of the nuclear interaction (see Cugnon et al. in Ref. 20).

For individual two body scatterings, what is actually done in the VUU computer program is to allow the particles to scatter and conserve energy as in the cascade model. Energy is also conserved for the ensemble average, but not within each separate ensemble because of the coupling between different ensembles. For a typical integration time step $\Delta t=0.25 \mathrm{fm} / c$, the final ensemble energy differs from the initial ensemble energy by $6 \%$.

The test particle also moves along a trajectory influenced by the gradient of the mean field and the occupation of the final state in a two particle scattering event. This approach thus goes beyond that of Wong et al. in Ref. 20 by including the Pauli principle and collisions. The trajectory functions $r, p$ are in the VUU model determined by the mean field, the Pauli principle, and two body collisions. The density function $\rho$ is evaluated from the distribution function to generate the self-consistent potential at each new time step. The interaction $U$ is of the effective Skyrme-type where the velocity dependence of the force is ignored. Here we mainly use the hard equation of state that has been successful in explaining collective flow and the pion yields. ${ }^{20}$ This, then, is a three-dimensional dynamic model of the nuclear collision and disassembly.

First we study a system of 40 nucleons initialized at a density $\rho=1.5 \rho_{0}$ and with different temperatures $T=5$, 10,15 , and $20 \mathrm{MeV}$ with a finite temperature FermiDirac distribution. The zero temperature Fermi gas initialization of the VUU model ${ }^{20}$ is thus changed to a finite temperature Fermi gas. Recall that for a finite temperature Fermi gas, the density of particles in momentum space is

$$
d n / d^{3} p=4 \Delta V /(2 \pi \hbar)^{3}\left(e^{(E-\mu) / T}+1\right),
$$

where $E$ is the total energy and $\mu$ is the chemical potential. The initial compression $\rho / \rho_{0}=1.5$ and temperature $T$ uniquely determine a chemical potential. This momentum distribution is given to the test nucleons and they are assigned positions in configuration space within a Fermi sphere.

The system is then allowed to expand according to the

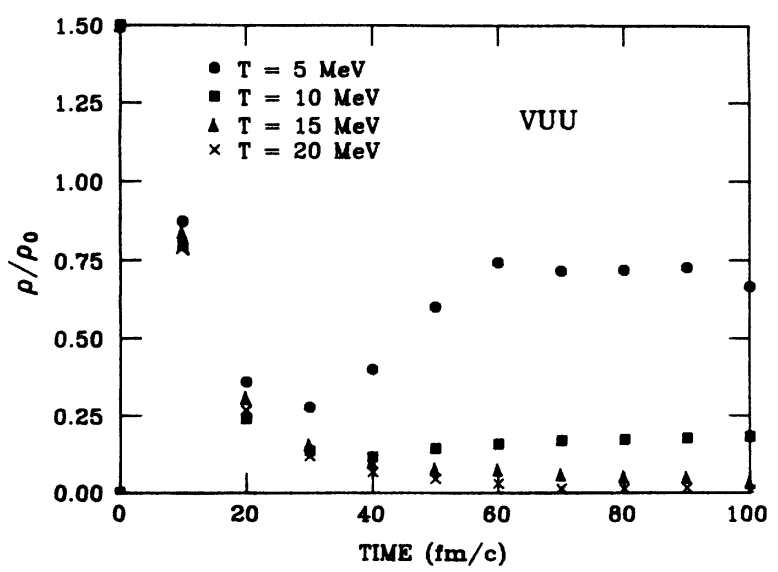

FIG. 1. Density around the origin for an expanding initially compressed $\left(\rho / \rho_{0}=1.50\right)$ system at $T=5,10,15$, and $20 \mathrm{MeV}$ initial temperatures in the VUU model. 
VUU model. Even though the collision term is zero in the initial equilibrium situation, the higher initial kinetic energy (from the finite temperature Fermi-Dirac momentum distribution) and the repulsive mean field drive the system apart and there are scatterings as the fireball expands.

Even though the VUU approach includes binding through the mean field, it is still a nontrivial problem to extract the distribution of fragments and temperatures. We therefore study the time evolution of the density in a central region of radius $3 \mathrm{fm}$ (Fig. 1). Just as in finite temperature Hartree-Fock (FTHF) calculations, ${ }^{21}$ the density rapidly decreases with the temperature. Observe that for temperatures $T \geq 10 \mathrm{MeV}$, there is no central residue formed. However, for $T=5 \mathrm{MeV}$, the mean field is still able to bind the nucleons together: from $t=30$ $\mathrm{fm} / c$ to $60 \mathrm{fm} / c$, nuclear matter is attracted back into the central region (see Fig. 1). But at higher initial temperatures, the larger momentum spread causes rapid expansion and overstress: the density quickly falls to less than $\rho_{0}$ throughout space. The expansion here would not necessarily be isentropic, as many fluid dynamic models assume, since we have neither the collisionless limit of mean field theory nor are collisions so frequent as to maintain local thermal equilibrium.

FTHF calculations ${ }^{21}$ for $T<5 \mathrm{MeV}$ take the influence of excitation energy of the nucleus into account. However, FTHF is equilibrium thermodynamics without an explicit treatment of the evaporation of nucleons. Here we have applied the VUU method for $T \geq 5 \mathrm{MeV}$ where the occupation probabilities are significant for unbound states. The evaporation of nucleons into the continuum is thus treated.

At normal conditions, nuclear matter is a Fermi liquid, being in a metastable superheated state for moderate temperatures. The density of nuclear matter decreases with $T$, whereas both the energy per particle and the entropy naturally increase. ${ }^{21}$ Unlike in low temperature FTHF calculations, we see in the VUU approach that the density of nuclear matter does not tend to vanish outside of the nucleus: some of the expanding matter must form fragments. In fact, for the highest temperatures, there is no nucleus, all of the nucleons are evaporated.

We have side-stepped the quantum mechanical question of fragment formation by looking at the density. In order to have a rough idea of the fragment mass distribution, one could look at the density throughout phase space and assign a mass number to a fragment based upon that density. This has been done by others. ${ }^{8} \mathrm{We}$ prefer not to do this for two reasons: (1) in some regions of phase space, there will then be a fraction of a fragment, (2) one will then also have to model the quantum mechanical decay of fragments. Models of the VUU type are thus presently unable to provide a believable distribution of final state fragments.

What can be expected for higher initial compressions and temperatures is that the system will rapidly disassemble. For low excitation energies or temperatures the system of nucleons would yield a highly excited compound nucleus or binary fragmentation. At the highest temperatures studied here, we clearly see a transition to a gase- ous phase where the average interparticle distance is much larger (Fig. 1).

Calculations done by others ${ }^{22}$ for the Vlasov equation and with the full VUU equation are consistent with the above results. At low temperature, small compressions simply result in the oscillation of the nucleus and the slow evaporation of particles. Neutral nuclei break up even at $T=0$ for $\rho \gg \rho_{0}$. The conclusions do not change appreciably when a Coulomb interaction and an isospin dependent term is added to the mean field. ${ }^{22}$ For an isolated compressed nucleus, the effect of the collision term is to cause particle loss to be somewhat larger at low initial densities.

The situation of interest is that of an actual heavy ion collision, which is not spherically symmetric. In Fig. 2 we show the density in a central region of radius $2 \mathrm{fm}$ from a simulation using the VUU model for $\mathrm{Au}+\mathrm{Au}$ at two different energies $E_{\text {lab }}=50$ and $250 \mathrm{MeV} /$ nucleon, respectively. Values for the density obtained with a larger radius of $3 \mathrm{fm}$ differ by less than $10 \%$. Furthermore, the time evolution of this central density is practically identical for $1 \mathrm{fm}<b<7 \mathrm{fm}$; for more peripheral collisions, the maximum density will, of course, fall. Note in Fig. 2 the same transition behavior of the time dependence of the central density as in the ideal spherical case (Fig. 1). At the lower energy (50 MeV/nucleon), the attractive part of the mean field is still able to bind a central residue, whereas at the higher energy none is left.

The slope parameters $T_{0}$ extracted from the final state invariant proton cross sections

$$
\sigma_{I}=E d^{3} \sigma / d p^{3}=d^{2} \sigma / p d K d \Omega
$$

at $90^{\circ}$ (by assuming that $\sigma_{I} / E=e^{-K / T_{0}}$ ) are equal to 19 and $35 \mathrm{MeV}$, respectively. The actual temperature achieved in a central region will be less than these values. $^{2,23}$ For example, the $19 \mathrm{MeV}$ exponential tail can be attributed largely to the finite $\left\langle p^{2}\right\rangle$ whereas within a real fragment $\langle p\rangle=0$.

One may also obtain a classical temperature from the

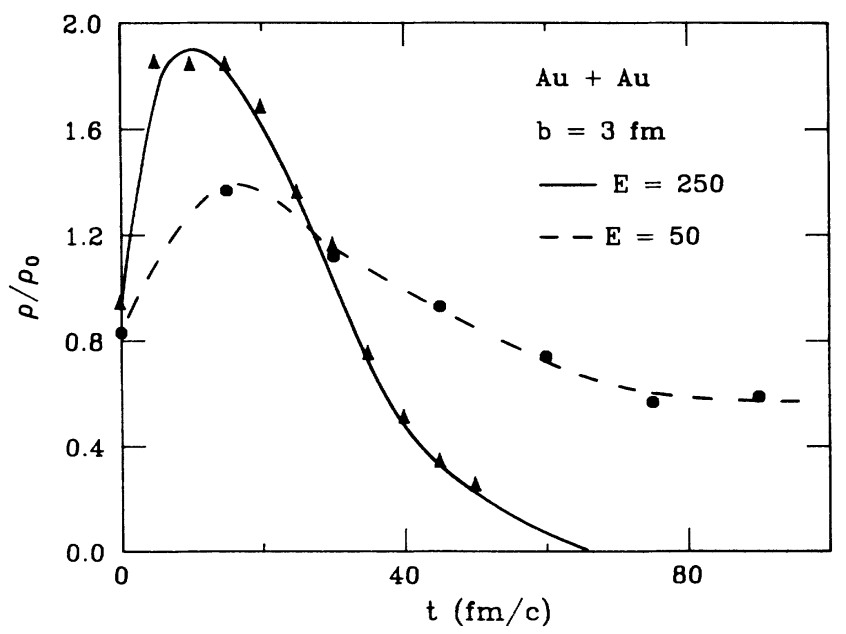

FIG. 2. Central density vs time for $\mathrm{Au}+\mathrm{Au}$ at $b=3 \mathrm{fm}$ and $E_{\text {lab }}=50$ and $250 \mathrm{MeV} /$ nucleon in the VUU model. 


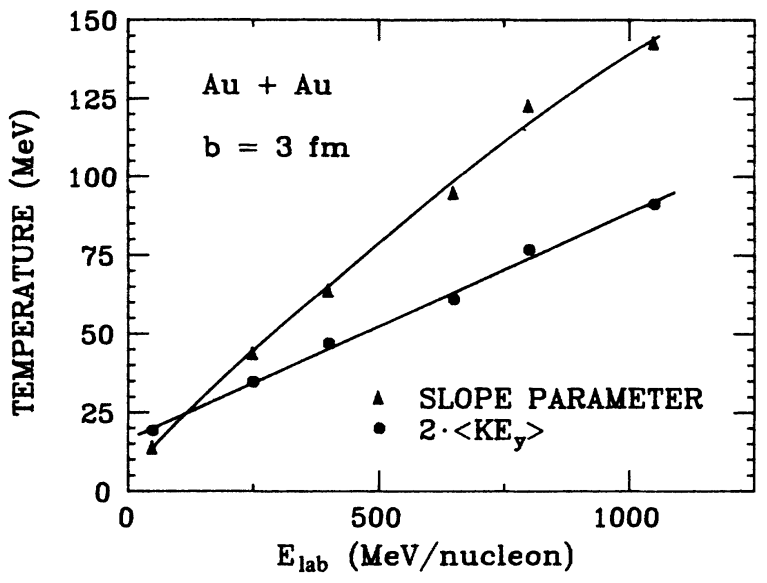

FIG. 3. Temperature from $\mathrm{Au}+\mathrm{Au}$ collisions in the VUU model extracted from the invariant cross section at $90^{\circ}$ and from the kinetic energy of a central region.

equipartition theorem: the kinetic energy from the $x$ and $y$ components of the nucleons momentum within a small central sphere of radius $2 \mathrm{fm}$ may then be associated with a temperature. This temperature then rises from zero (since there are no particles in the central region initially) to a maximum value and then falls again. We find that the maximum of $\left\langle p_{x}^{2} / 2 m\right\rangle$ is less than $15 \%$ larger than $\left\langle p_{y}^{2} / 2 m\right\rangle$ at all the energies studied. Since there is naturally some flow energy (mainly due to $p_{x}$ ), we simply assign a $T$ value based upon $2\left\langle p_{y}^{2} / 2 m\right\rangle$.

We show in Fig. 3 the difference between the slope parameter and this maximum classical temperature for $\mathrm{Au}+\mathrm{Au}$ collisions from $E_{\text {lab }}=50$ to $1050 \mathrm{MeV} /$ nucleon in the VUU model. Note that such a classical temperature of the equilibrated central region is generally less than the slope parameter. We thus see in Fig. 3 the important result that the spectrum of emitted nucleons is

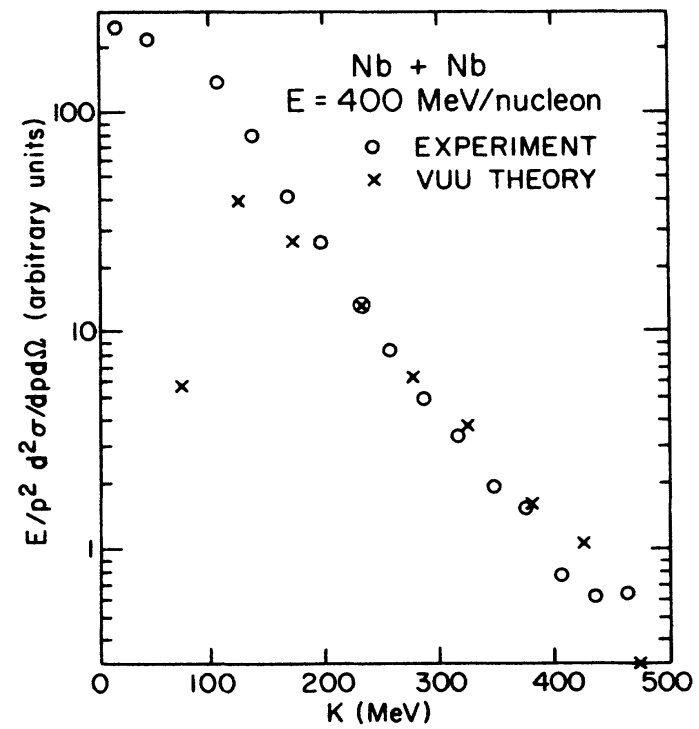

FIG. 4. Invariant cross section at $90^{\circ}$ for $\mathrm{Nb}(400$ $\mathrm{MeV} /$ nucleon $)+\mathrm{Nb}$ in the VUU model and experimentally from the Lawrence Berkeley Laboratory/Gesellschaft für Schwerionenforschung plastic ball group. much hotter than the kinetic energy spectrum of the central emitting region (see also Vicentini et al. in Ref. 9 for a discussion of the problem from the point of view of molecular dynamics). This classical kinetic energy does not give the "true" temperature since classically a $T=0$ Fermi gas has an apparent temperature because of the nonzero kinetic energy.

The experimental slope parameters for a variety of systems range from $25 \mathrm{MeV}$ (at $200 \mathrm{MeV} /$ nucleon) to 90 $\mathrm{MeV}$ (at $1050 \mathrm{MeV} /$ nucleon). ${ }^{2}$ For the reaction $\mathrm{Nb}(400$ $\mathrm{MeV} /$ nucleon) $+\mathrm{Nb}$, we compare in Fig. 4, the experimental data ${ }^{24}$ (for high multiplicity) to the results of the VUU model at $b=3 \mathrm{fm}$ impact parameter. Note the exceptional agreement for the high energy tail. The experimentalists find $T_{0}=65 \mathrm{MeV}$ whereas the VUU model predicts a value of $70 \mathrm{MeV}$. The low kinetic energy part of the distribution might be better reproduced by averaging over impact parameters. Such extracted $T_{0}$ values represent an upper limit for the temperature.

We have studied in more detail the $\mathrm{Nb}+\mathrm{Nb}$ system at various energies from 50 to $1050 \mathrm{MeV} /$ nucleon (Fig. 5). The time and impact parameter dependence of $\mathrm{Nb}+\mathrm{Nb}$ collisions before this late time has been discussed else-

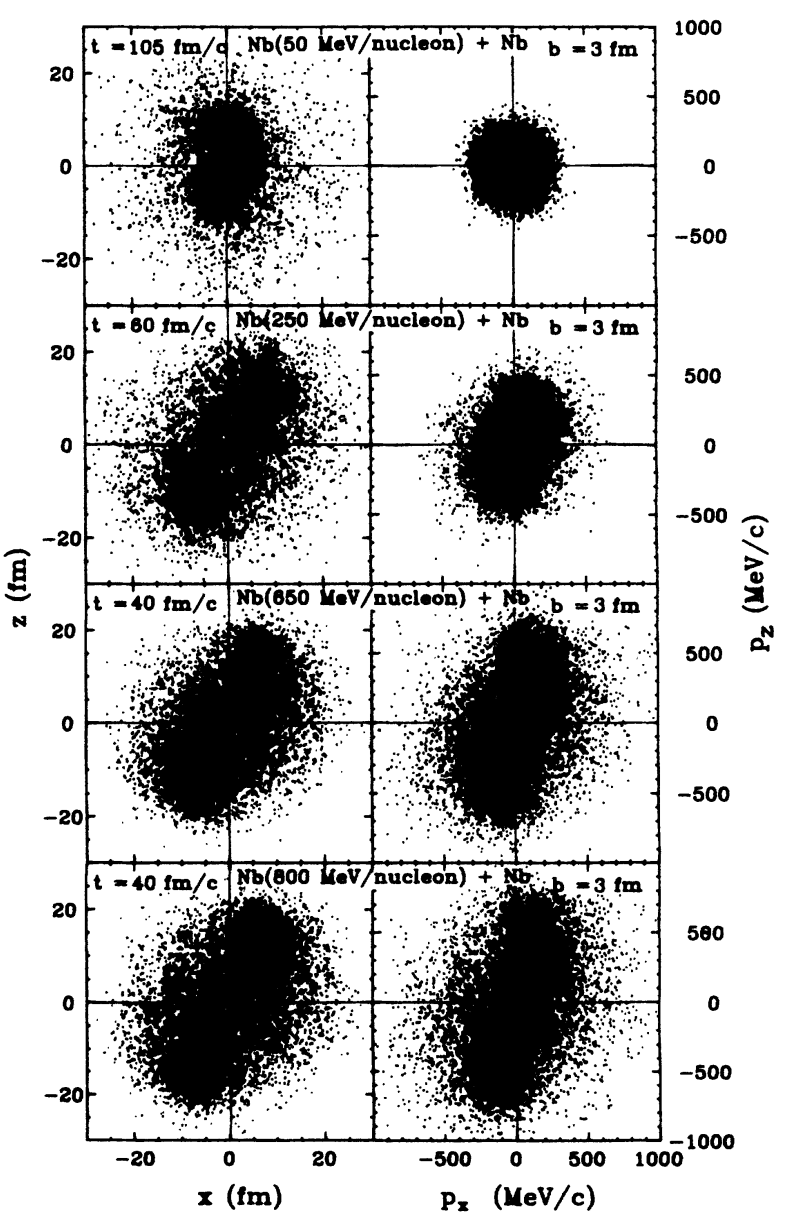

FIG. 5. The results of 30 ensembles are superposed in order to represent the distribution function in the final state in configuration and momentum space for $\mathrm{Nb}+\mathrm{Nb}$ collisions at different energies. 


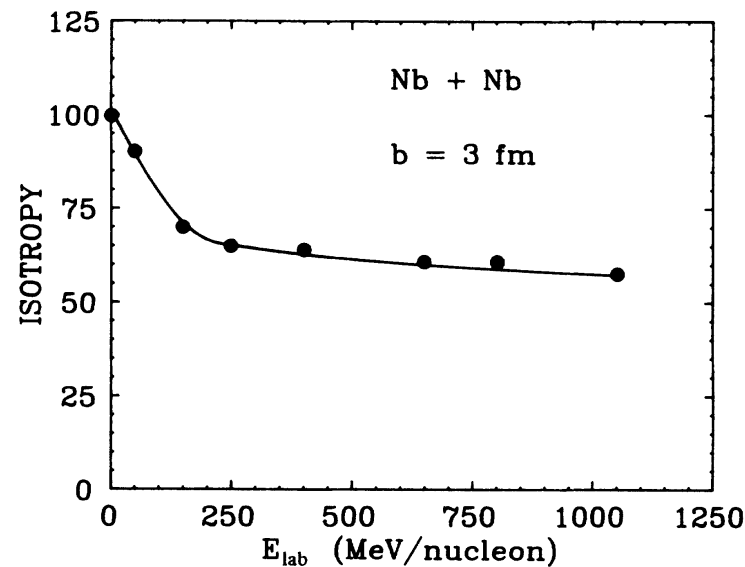

FIG. 6. Isotropy vs energy for all the nucleons for $\mathrm{Nb}+\mathrm{Nb}$ collisions in the VUU approach.

where. $^{25}$ In Fig. 5 we show projections of the Wigner function into the $x-z$ plane in configuration and momentum space for the final state. In the initial state, the target and projectile resided within their separate Fermi spheres. Observe that at the lowest energy, there is some central residue, just as was seen in the $\mathrm{Au}+\mathrm{Au}$ case (Fig. 2).

The momentum distribution in the final state is basically spherical for $\mathrm{Nb}+\mathrm{Nb}$ at $50 \mathrm{MeV} /$ nucleon. This can be seen from using the ratio of transverse to longitudinal momenta

$$
R=2 / \pi \Sigma p_{\perp} / \Sigma p_{\|},
$$

where $p_{\perp}$ and $p_{\|}$are the momenta perpendicular to and parallel to the beam $(z)$ direction. This global quantity is plotted versus the laboratory energy in Fig. 6.

The equilibration of the heavy remnant (see Fig. 5) is the result of a complicated interplay between the mean field, the Uehling-Uhlenbeck collision term, and the Pauli principle. At the lowest energies, the Uehling-Uhlenbeck collision term results in strong global equilibration, just as for the lighter $\mathrm{Ar}+\mathrm{Ca}$ system. ${ }^{26} \mathrm{~A}$ substantial degrading of the initial momenta occurs due to the collision term. ${ }^{26}$ At the energy $E=1050 \mathrm{MeV} /$ nucleon, $R$ does not decrease drastically indicating equilibration of the participants and some stopping even at this high energy. A softer equation of state would result in less isotropy, e.g., smaller $R$ values, because of the smaller repulsion from the high density interaction region. The decrease of $R$ for the participants alone may be less than indicated by Fig. 6 because we have calculated $R$ globally: the spectators have greater $p_{\|}$at the higher energies. With the Pauli blocking turned off, the $R$ values would increase due to the shorter mean free path.

If one analyzes the transverse momentum distribution, one finds a negative value for the transverse momentum transfer at $50 \mathrm{MeV} /$ nucleon. $^{27}$ Experimentally, light particles have been found to be emitted preferentially to negative angles. ${ }^{28}$ The attractive part of the nuclear potential and surface effects are dominant at this low energy. As the energy is increased, the final state momentum distribution becomes less isotropic (Figs. 5 and 6). There is no central residue formed, the target and projectile completely disintegrate. Also, the matter at projectile rapidity receives a positive transverse momentum transfer. ${ }^{27}$ There is thus a change in sign of the momentum transfer for the $\mathrm{Nb}+\mathrm{Nb}$ system at around $100 \mathrm{MeV} /$ nucleon.

Thus in heavy ion collisions, what one observes experimentally as the energy increases is the loss of the ability of the mean field to bind the hot expanding nuclear matter and a change in the sign of the momentum transfer from negative to positive. Certainly, not every heavy ion interaction does result in such transition behavior. Peripheral collisions deposit little energy and result in a disassembly process via evaporation of nucleons and composites. Peripheral collisions also result in little momentum transfer from the nuclear equation of state.

In summary, we have studied the expansion of a hot compressed system both from a Fermi-Dirac distribution and in simulated heavy ion collisions. The mean field of the VUU model exhibits a binding property that changes with bombarding energy. Not only does the transverse momentum transfer change from negative to positive but also the nature of the central residue changes. More theoretical and experimental work is necessary to understand this transition behavior.

We express our appreciation to the Michigan State University National Superconducting Cyclotron Laboratory, the Institute for Theoretical Physics in Frankfurt, and to the Academic Computing Center at Muhlenberg College.
*Present address: Sektion Physik, Universitat Munchen, D8046 Garching, Federal Republic of Germany.

†Present address: Physics Department, University of California, Berkeley, CA 94720.

${ }^{1}$ P. Danielowicz, Nucl. Phys. A314, 465 (1979).

${ }^{2}$ H. Stoecker, G. Buchwald, G. Graebner, P. Subramanian, J. A. Maruhn, W. Greiner, B. V. Jacka, and G. D. Westfall, Nucl. Phys. A400, 63 (1983).

${ }^{3}$ G. Bertsch and P. J. Siemens, Phys. Lett. 126B, 9 (1983).

${ }^{4}$ J. A. Lopez and P. J. Siemens, Nucl. Phys. A431, 738 (1984).

${ }^{5}$ H. Schulz, B. Kaempfer, H. W. Borz, C. Ruepke, and J. Bondorf, Phys. Lett. 147B, 17 (1984).
${ }^{6}$ A. Bonasera and M. Di Toro, Lett. Nuovo Cimento 44, 172 (1985).

${ }^{7}$ M. E. Fisher, Phys. 3, 255 (1967); Rep. Prog. Phys. 30, 615 (1967); For a discussion of the liquid-gas phase transition see P. J. Siemens, Nature 305, 410 (1983).

${ }^{8}$ J. Knoll and B. Strack, Phys. Lett. 149B, 45 (1984).

${ }^{9}$ W. Bauer, D. R. Dean, U. Mosel, and U. Post, Phys. Lett. 150B, 53 (1985); X. Campi and J. Desbois, Proceedings of the 7th High Energy Heavy Ion Study (Gesellschaft für Schwerionforschung, Darmstadt, 1984); A. Vicentini, G. Jacucci, and V. R. Pandharipande, Phys. Rev. C 31, 1783 (1985). 
${ }^{10}$ H. R. Jaqaman, A. Z. Mekjian, and L. Zamick, Phys. Rev. C 29, 2067 (1984).

${ }^{11}$ J. P. Bondorf, R. Donangelo, H. Schulz, and K. Sneppen, Phys. Lett. 162B, 30 (1985).

${ }^{12}$ A. S. Hirsch, A. Bujak, J. E. Finn, L. J. Gutay, R. W. Minich, N. T. Porile, R. P. Scharenberg, B. C. Stringfellow, and F. Turkot, Phys. Rev. C 29, 508 (1984).

${ }^{13}$ H. Gutbrod, A. Warwich, and H. Wieman, Nucl. Phys. A387, 177 (1982).

${ }^{14}$ M. W. Curtin, H. Toki, and D. K. Scott, Phys. Lett. 123B, 289 (1983); A. D. Panagiotou, M. W. Curtin, H. Toki, D. K. Scott, and P. J. Siemens, Phys. Rev. Lett. 52, 496 (1984).

${ }^{15}$ H. Machner, Phys. Rev. C 31, 1271 (1985).

${ }^{16}$ R. Trockel, K. D. Hildebrand, U. Lynen, W. Mueller, H. G. Rabe, H. Sann, H. Stelzer, R. Wada, N. Brummund, R. Glasow, K. H. Kampert, R. Santo, D. Pelte, J. Pochadzalla, and E. Eckart, Gesellschaft für Schwerionenforschung Report 85-45; J. B. Cumming, Phys. Rev. C 32, 1445 (1985).

17J. Gosset, J. I. Kapusta, and G. Westfall, Phys. Rev. C 18, 844 (1978); P. R. Subramanian, L. P. Csernai, H. Stoecker, J. A. Maruhn, W. Greiner, and H. Kruse, J. Phys. G 47, 241 (1981).

18J. Aichelin and H. Stoecker, Phys. Lett. 163B, 59 (1985).

19J. Cugnon, D. Kinet, and J. Van der Meulen, Nucl. Phys. A379, 553 (1982); J. J. Molitoris, H. Stoecker, H. A. Gustafsson, J. Cugnon, and D. L'Hote, Phys. Rev. C 33, 867 (1986).

${ }^{20}$ H. Kruse, B. V. Jacak, and H. Stoecker, Phys. Rev. Lett. 54, 289 (1985); J. J. Molitoris and H. Stoecker, Phys. Rev. C 32,
346 (1985); J. J. Molitoris, H. Stoecker, and B. L. Winer, ibid. 36, 220 (1987); for similar approaches see C. Y. Wong, ibid. 25, 1460 (1982); G. Bertsch, H. Kruse, and S. dasGupta, ibid. C 29, 673 (1984); B. Remaud, F. Sebille, C. Gregoire, and F. Scheuter, Nucl. Phys. A428, 101c, 1984; J. Cugnon, A. Lejeune, and P. Grange, Phys. Rev. C 35, 861 (1987).

${ }^{21}$ G. Sauer, H. Chandra, and U. Mosel, Nucl. Phys. A264, 221 (1976); G. Bozzolo and J. P. Vary, Phys. Rev. C 31, 1909 (1985).

${ }^{22}$ G. E. Beauvais, D. H. Boal, and J. C. K. Wong, Phys. Rev. C 35, 545 (1987); G. E. Beauvais and D. H. Boal, University of Illinois Report P/86/2/26; R. J. Lenk and V. R. Pandharipande, Phys. Rev. C 34, 177 (1986).

23J. Pochadzalla et al., Phys. Lett. 161B, 275 (1985).

${ }^{24}$ H. H. Gutbrod, M. Doss, H. A. Gustafsson, B. Kolb, H. Loehner, B. Ludewigt, A. M. Poskanzer, H. G. Ritter, and H. Wieman, Lawrence Berkeley Laboratory report, 1985.

${ }^{25}$ J. J. Molitoris and H. Stoecker, Phase Space Approach to $\mathrm{Nu}$ clear Dynamics (World-Scientific, Singapore, 1986).

${ }^{26}$ H. Kruse, B. V. Jacak, J. J. Molitoris, G. D. Westfall, and H. Stoecker, Phys. Rev. C 31, 1770 (1985).

${ }^{27}$ J. J. Molitoris, D. Hahn, and H. Stoecker, Nucl. Phys. A447, 13c (1985); J. J. Molitoris and H. Stoecker, Phys. Lett. 162B, 47 (1985).

${ }^{28}$ M. B. Tsang, R. M. Ronningen, G. Bertsch, Z. Chen, C. B. Chitwood, D. J. Fields, C. K. Gelbke, W. G. Lynch, T. Nayak, J. Pochadzalla, T. Shea, and W. Trautmann, Phys. Rev. Lett. 57, 559 (1986). 